\title{
Spotty scope and our relation to fictions 1
}

\author{
Tim Button
}

\begin{abstract}
Copyright. This paper is due to appear in Noûs. This paper may be subject to minor
\end{abstract} changes. The authoritative version should be obtained from Nô̂s

\begin{abstract}
Whatever the attractions of Tolkein's world, irrealists about fictions do not believe literally that Bilbo Baggins is a hobbit. Instead, irrealists believe that, according to The Lord of the Rings \{Bilbo is a hobbit\}. But when irrealists want to say something like "I am taller than Bilbo", there is nowhere good for them to insert the operator "according to The Lord of the Rings". This is an instance of the operator problem. In this paper, I outline and criticise Sainsbury's 2006 spotty scope approach to the operator problem. Sainsbury treats the problem as syntactic, but the problem is ultimately metaphysical.
\end{abstract}

Irrealists about fictions wish to talk about fictions without committing themselves to the literal truth of those fictions. To distance themselves from the literal truth of a fiction, they might employ a fictional operator, such as "according to the fiction". For example, instead of saying "Bilbo is a hobbit", irrealists might say "according to The Lord of the Rings \{Bilbo is a hobbit\}". But when irrealists want to say something like "I am taller than Bilbo", there is nowhere good to insert the operator "according to The Lord of the Rings". This is an instance of a well known problem for irrealists, which I call the operator problem.

Given the difficulties that arise for irrealists when they use operators, one might think that irrealists could handle fictitious scopes using something syntactically more sophisticated than operators. To this end, Sainsbury (2006) has proposed that we allow fictional contexts to have spotty scope. However, the operator problem is ultimately a metaphysical problem. Accordingly, Sainsbury needs to supply a semantics for his notion of spotty scope, to show that it is acceptable to irrealists. Sainsbury does not do this, and I argue that he cannot. More generally, I suggest that no new theory of fictional contexts will solve the operator problem.

\section{The operator problem}

Irrealists want to distance themselves from treating fictions as literally true. They do not want to incur any serious ontological or epistemological costs by talking about fictions or fictional characters. They do not think that anything is a fictional object. Literally speaking, they do not think that there are hobbits, or orcs, or magical rings, whatever LotR (The Lord of the Rings) says. They do not think that we can refer to fictional objects, since there are none. ${ }^{1}$

Irrealism is a very sensible thesis. If any area of discourse is not literally true, and incurs neither ontological commitments nor serious epistemological baggage, discourse about fictions is a good candidate. But irrealism is not always easy to maintain. An initial difficulty arises because irrealists think that fictional names, like "Bilbo" and "Gandalf", are referentless. It is common to treat simple sentences that contain referentless names as (in some sense) vacuous, ${ }^{2}$ but intuitively, the following sentences are true rather than vacuous: 


\section{Bilbo is a hobbit.}

\section{Bilbo is shorter than Gandalf.}

To handle this, irrealists will have to invoke some fictitious context, within which these sentences are not vacuous. Of course, irrealists will want not just one fictional context, but many (probably at least one fictional context per fiction). Irrealists will also want to be able to embed different fictional contexts within each other, to handle fictions within fictions.

A natural way for irrealists to handle fictional contexts is in terms of fictional operators. The canonical operator is "according to the fiction $\{\ldots\}$ ". The idea is that, when " $\varphi$ " is fictionally true, an irrealist can assert "according to the fiction $\{\varphi\}$ " rather than simply asserting " $\varphi$ ”. Operators are attractive to irrealists, because the use of an operator justifies the irrealist's claim that she is not committed to $\varphi$. Her only commitment is that " $\varphi$ " is true in some fiction. For example, an irrealist does not that there is a hobbit called "Bilbo"; she just thinks that, according to LotR \{there is a hobbit called "Bilbo"'

It is natural to treat operators as prefixes (i.e. monadic sentential connectives). Unfortunately, this immediately gives rise to a well known problem. ${ }^{3}$ Standing at over 6'6", my friend Adam is a very tall man. Bilbo Baggins is a regular-sized hobbit from Middle Earth. So:

1. Adam is taller than Bilbo.

Irrealists do not believe that Bilbo literally exists, but they do want to treat (1) as true. So, can the irrealist avoid having to believe in hobbits by inserting an operator into [1]? She might try:

1. according to LotR $\{$ Adam is taller than Bilbo

But this makes Adam into an inhabitant of Middle Earth, which he isn't. Instead, the irrealist might try:

$$
\mathbf{1}]^{\prime} . \exists x \text { (Adam is taller than } x \text { and according to } \operatorname{Lot} R\{x=\text { Bilbo }\} \text { ) }
$$

But then the variable " $x$ " falls both inside and outside the fiction operator, making Bilbo both fictional and real. (To be sure, there are cases in which variables can legitimately fall on both sides of a fictional operator-Charles Dickens wrote novels about London-but this is not one of those cases.)

There is nowhere else for the operator to go. Irrealists therefore apparently cannot use operators to handle (1). This is the operator problem for irrealists.

Sentence (1) will serve as my main example of the operator problem. But, rather worryingly, instances of the problem can be generated schematically. The face-value reading of (1) is a sentence of the form " $a R$ s $b$ ", where " $a$ " names something real, " $b$ " names something fictional, and " $R$ " names some relation that holds between them; and it is clear that any sentence of this form is an instance of the operator problem. Moreover, by allowing both names to name fictional characters from different fictions, we can generate inter-fictional instances of the operator problem, such as: 
2. Bilbo is taller than Thumbelina.

The problem here is just that that neither the novel LotR, nor the fairy-tale Thumbelina, contains both Bilbo and Thumbelina.

In this paper, I shall only consider the difficulties that the operator problem causes for irrealists. Perhaps realists also face some version of the operator problem, but I shall not consider that here. ${ }^{4}$ I just want to determine whether irrealists can weather the storm.

\section{Two quick responses}

There are various possible responses to the operator problem. In this section, I shall mention two of them. They are not the focus of the paper, so I shall not explore them in depth. However, it is important to understand them now, so that I can invoke them later. (In particular, I shall want to show that Sainsbury's apparently novel "spotty scope" proposal is in danger of collapsing into one of these two approaches.)

Austere-irrealism. The first response to the operator problem runs thus. The irrealist concedes that (1) is literally false, for (1) suggests that Bilbo Baggins exists, and he doesn't. The irrealist also concedes that this is problematic, since we are tempted to assert things like (1). But, our irrealist continues, (1) is tempting just because it gets at a nearby sentence, which is both acceptable for irrealists and literally true. For example, our irrealist might claim that $(1)$ is getting at: ${ }^{5}$

17. $\exists x$ (Adam is over $x$ inches tall, and according to $\operatorname{Lot} R$ (Bilbo is under $x$ inches tall $\}$ )

This sentence is clearly true: Adam is over 78 inches tall, whereas according to $\operatorname{LotR}$ \{Bilbo is under 78 inches tall\}. Moreover, $(1 \mathrm{l})$ seems to uncover our reasons for wanting to assert $(1)$ in the first place: if we don't think that Bilbo is under 78 inches tall (according to LotR), then we have no reason for wanting to accept anything like (1). This response also has the virtue of austerity. It uses no new resources to deal with the operator problem, beyond a quite ordinary fictional operator, "according to $\operatorname{Lot} R\{\ldots\}$," which the irrealist uses even to deal with simple sentences like "Bilbo is a hobbit". For this reason, I call this strategy austere-irrealism. ${ }^{6}$

The austere-irrealist has an excellent way to deal with (1) and, clearly, the austere-irrealist can offer a very similar treatment of (2), i.e.:

2a. $\exists x$ (according to LotR $\{$ Bilbo is over $x$ inches tall $\}$, and according to Thumbelina \{Thumbelina is under $x$ inches tall $\}$ )

However, the austere-irrealist may not want to offer this treatment for every operator problem which invokes a comparative relation. The problem is that this treatment incurs a commitment to degrees of comparison. In the case of is taller than, this seems perfectly reasonable: heights come by degrees, since they can be measured, with a ruler, in inches. But consider other comparisons, like: 
3. Adam is wittier than Odysseus.

4. Adam is more affable than Ajax.

If we want to treat these sentences in the same way, we will be committed to degrees of wittiness and affability. This may seem slightly odd.

More generally, plenty of operator problems do not involve comparisons at all. For example: ${ }^{7}$

5. Adam pities Bilbo.

It is doubtful that this sentence gets at any nearby sentence which only mentions properties that Adam has and properties such that according to LotR \{Bilbo has those properties\}. Equally, I doubt that we can limit our attention to properties such that according to The Iliad \{Odysseus has those properties\} and properties such that according to Inferno \{Ulisse has those properties\}, if we want to deal with:

6. Odysseus (of The Iliad) is the same person as Ulisse (of Dante's Inferno, Canto 26).

The austere-irrealist will have to look for more exotic treatments of these operator problem sentences.

Incorporating-irrealism. A second appealing response for irrealists runs thus. The primary difficulty with $(1)$ is that no single fiction contains both Adam and Bilbo. To rectify this, we could surely create some such wider fiction. We could then offer: ${ }^{8}$

1. according to extended-LotR $\{$ Adam is taller than Bilbo $\}$

Evidently, we could deal with 2 in the same way, just by creating a single fiction LotR+Thumbelina, which contained both characters from Middle Earth and Thumbelina. Since this strategy involves incorporating elements from all over the place into a single, wide fiction, I call this incorporating-irrealism.

One immediate difficulty with this strategy is that it seems to multiply problems. We now need to explain both how real-Adam relates to extended-LotRAdam, and how LotR-Bilbo relates to extended-LotR-Bilbo. A natural explanation is that real-Adam and extended-LotR-Adam have exactly the same height property, and likewise for LotR-Bilbo and extended-LotR-Bilbo. But this is surely just to say:

$1\}^{\prime} . \exists x$ (Adam is over $x$ inches tall and according to extended-Lot $R$ Adam is over $x$ inches tall $\}$ and according to $\operatorname{Lot} R\{$ Bilbo is under $x$ inches tall $\}$ and according to extended-Lot $R$ (Bilbo is under $x$ inches tall $\}$ ) 
which is scarcely more useful than the austere-irrealist's (1a). At some level, then, it unclear that incorporating-irrealism and austere-irrealism differ tremendously. For related reasons, it is not immediately clear how the incorporatingirrealist should handle sentences $(3-6){ }^{9}$

To repeat myself: there is much more to be said about both austere-irrealism and incorporating-irrealism. I have no wish to pass judgement on either approach here. But I do want to emphasise the intrinsic appeal of both strategies. Both strategies only involve fictional operators. We assumed at the outset that it was acceptable for irrealists to use operators to distance themselves from fictions. So both strategies, if they are successful, are clearly acceptable for irrealists. This is why they are worth exploring.

Nonetheless, I shall set these strategies aside for now, and move on to the main purpose of this paper: to criticise Sainsbury's (2006) "spotty scope" solution to the operator problem. To be clear about my target: Sainsbury has offered a tremendous contribution to the philosophy of fiction in his (2005) and (2010), but he does not discuss "spotty scope" in either book. Accordingly, I shall treat Sainsbury's "spotty scope" proposal in isolation from the rest of his work, and all further mention of "Sainsbury" should be read as "Sainsbury-as-of-2006" (unless explicitly stated otherwise).

\section{Sainsbury's spotty scope logic}

Fictional operators are syntactically clunky. Any given atomic sentence must either fall entirely inside an operator, or entirely outside an operator. But in ordinary parlance, we dip in and out of fictitious scopes much more freely than operators allow. This seems to be what causes the operator problem.

Accordingly, Sainsbury responded to the operator problem by inventing a new syntactic tool for indicating fictional contexts: underlining. For instance, where we are to interpret underlining as delimiting the scope of Lot $R$, an irrealist might offer sentences like: ${ }^{10}$

\section{Bilbo is a hobbit.}

\section{Bilbo is shorter than Gandalf.}

and, in the case of operator problem sentences:

1;. Adam is taller than Bilbo.

The virtue of underlining, of course, is that it is syntactically freer than an operator. We can underline only a part of a sentence, as in $(1 ;)$, and we can continue to dip in and out of fictional scope at will:

..., and Bilbo is a hobbit, and Adam pities hobbits.

A suitably regimented formal language, with multiple levels of underlining and overlining to introduce different fictional scopes, could be a useful tool for dis- 
cussing fictions. For example, we could handle the following (intuitively legitimate) argument:

15. Adam is taller than Bilbo.

23. Bilbo is taller than Thumbelina.

$\therefore$ Adam is taller than Thumbelina.

Likewise, we could talk about fictions within fictions, by stacking multiple levels of underlining and overlining together. Call the first-order logic that is augmented with a countable infinity of distinct underlining/overlining marks "SSL", for spotty scope logic.

Over the remainder of this paper, I shall consider whether irrealists can use SSL to solve the operator problem. My main objection is as follows. Sainsbury has invented a new syntactic tool without supplying it with a semantics. However, when SSL is given a semantics, irrealists are barred from employing SSL.

\section{Branched Quantifiers and Spotty Scope}

Irrealists face an essentially metaphysical problem: they don't want to incur ontological and epistemological commitments by postulating that there are hobbits, but they do want to believe that Adam is taller than one of them. Sainsbury seems to have responded to this metaphysical problem by adopting a new logic. This might come across as ad hoc. To meet this objection, Sainsbury aims to supply independent motivations for adopting SSL. He argues that SSL is supported by a deep analogy between SSL and the logic of branched quantifiers. In this section, I shall explain his analogy, and demonstrate that Sainsbury is mistaken.

Sainsbury asks us to consider the following sentence: ${ }^{11}$

7. Some relative of each villager and some relative of each townsman hate each other.

It is to be understood that "villager" and "townsman" are reversible in (7). That is, the choice of one pair of relatives is independent from the choice of the other pair of relatives. (I take no stance on whether this intended reading is the sentence's natural reading.) There are only two likely candidates for formalising (7) in standard first-order logic:

$$
\begin{aligned}
& \text { 7. } \forall x \exists y \forall s \exists t((V x \wedge T s) \rightarrow(R x y \wedge R s t \wedge H y t)) \\
& \text { 7 }^{\prime} . \forall s \exists t \forall x \exists y((V x \wedge T s) \rightarrow(R x y \wedge R s t \wedge H y t))
\end{aligned}
$$

where " $V x$ " means that the denotation of " $x$ " is a villager, " $T x$ " means that the denotation of " $x$ " is a townsman, " $R x y$ " means that the denotation of " $x$ " is a relative of the denotation of " $y$ ", and "Hxy" means that the denotation of " $x$ " and the denotation of " $y$ " hate each other. But in standard first-order logic, 
we instantiate quantifiers in a linear fashion, working from left to right. So in the first sentence, the choice of townsman and relative depends upon the prior choice of villager and relative; and in the second, the choice of villager and relative depends upon the prior choice of townsman and relative. Neither eliminates the dependence, so neither provides the intended reading of (7).

To formalise the intended reading, we employ branched quantifiers. The syntactic trick is to stack quantifiers on top of one another to indicate that they fall outside each others' scope. So (7) becomes:

$$
\text { 7b. } \begin{aligned}
& \forall x \exists y \\
& \forall s \exists t
\end{aligned}((V x \wedge T s) \rightarrow(R x y \wedge R s t \wedge H y t))
$$

I shall call the logic which is augmented with branched quantifiers "BQL". Sainsbury suggests that we can instead capture the intended reading of (7) using his SSL:

$$
\text { 78. } \underline{\forall x \exists y} \overline{\forall s \exists t((V x \wedge T s) \rightarrow(R x y \wedge R s t \wedge H y t))}
$$

Again, the idea is that each quantifier-pair falls outside the scope of the other quantifier-pair, but the quantifier-free matrix falls within the scope of both pairs. Sainsbury goes on to claim that his SSL gains regressive support from its success in dealing with (7) and by its association with BQL:

The fact that spotty scope is found in a rigorous formal setting (branched quantifiers) should give us confidence in using its natural language analog in describing a variety of problematic natural language constructions. ${ }^{13}$

Sadly, Sainsbury is wrong: the notion of "spotty scope" invoked in BQL cannot be the notion of "spotty scope" invoked in SSL. The notion of "spotty scope" in SSL is not exactly clear. But the notion of "spotty scope" that is invoked in BQL concerns the scope of quantification, and only the scope of the quantification. When evaluating the truth value of a quantifier-free formula $\varphi$, with a given interpretation manual for each free variable in $\varphi$, ordinary first-order logic and BQL proceed in exactly the same way: they both use absolutely standard, modeltheoretic semantics (see the Appendix).

Problems now arise for Sainsbury because a sentence like (1) is already quantifierfree. As such, BQL has no resources for treating (1) beyond those available in standard first-order logic. Since standard first-order logic cannot handle (1), neither can BQL. But Sainsbury believes that we can use SSL to handle (1): all we need to do is underline judiciously, to generate (1\$). So if BQL cannot handle (1), whereas SSL can, then the two logics must operate with entirely different kinds of "spotty scope".

A more complicated example makes this point even clearer. Consider:

8. Some favourite hobbit of each villager and some favourite hobbit of each townsman hate each other.

This has exactly the same syntax as (7). Moreover, as with (7), we want it to be understood that "villager" and "townsman" are reversible in (8). So we must formalise 87 in the same way that we formalise 77 , i.e. as: 


$$
\text { 8b. } \quad \begin{aligned}
& \forall x \exists y \\
& \forall s \exists t
\end{aligned}((V x \wedge T s) \rightarrow(F x y \wedge F s t \wedge H y t))
$$

where "Fxy" means that the denotation of " $y$ " is a favourite hobbit of the denotation of " $x$ ". But in this case, the townsmen and villagers are real, whereas hobbits are not. So this is a BQL-instance of the operator problem. Taking a cue from Sainsbury, we might try to solve this instance of the problem by offering:

$$
\text { 8s. } \quad \forall x \underline{\exists y} \underline{\exists \underline{\exists}}((V x \wedge T s) \rightarrow(F x \underline{y} \wedge F s \underline{t} \wedge \underline{H y t}))
$$

But this is not a sentence of SSL (since the quantifiers are partially-ordered) and it is not a sentence of BQL (since it contains underlining). Evidently, then, BQL and SSL require utterly different notions of "spotty scope".

\section{Supplying a semantics for SSL}

Since SSL and BQL are distinct, spotty scope (in Sainsbury's sense) is not yet "found in a rigorous formal setting". The charge therefore remains that it is ad hoc to adopt SSL to solve the operator problem. That said, if SSL can solve the operator problem, that might be enough to recommend it to irrealists; they may simply take the charge of ad hocery on the chin. ${ }^{14}$

The truly important moral of the preceding section is simply that SSL requires a completely different kind of "spotty scope" from anything found in BQL. Accordingly, we cannot supply SSL with the same semantics as we supply BQL (see the Appendix for a sketch of the latter). So, what semantics could SSL receive? What is Sainsbury's notion of spotty scope? (It may be worth repeating the point flagged at the end of \$2 that Sainsbury's (2005) "reference without referents" framework offers no obvious help here.)

To see why SSL urgently needs a semantics, consider the following. In turning $\sqrt{1}$ into a sentence of SSL, I offered:

13. Adam is taller than Bilbo.

I might equally have offered:

$$
1 \boldsymbol{s}^{\prime} \text {. Adam is taller than Bilbo. }
$$

Both seem equally adequate in solving the operator problem. And, on reflection, this means that both are equally inadequate. (1\$) makes the is taller than relation part of the real world, whereas $\left[1 \beta^{\prime}\right)$ makes it fictional. But it is neither part of LotR, nor part of the real world, that Adam is taller than Bilbo. Rather, it is a feature of the $t w o$, arising from the heights of Adam (in the real world) and Bilbo (in LotR) respectively. However, this way of thinking about (1) would lead us to offer the following sentence of SSL:

1 $s^{\prime \prime} . \exists x$ (Adam is over $x$ inches tall and Bilbo is under $x$ inches tall) 
which is surely just a notational variant of the austere-irrealist's (11). So, in order to treat the spotty scope proposal as distinct from the previous irrealist proposals, we have to take seriously the idea that the is taller than relation bridges the gulf between reality and the fiction. Of course, we could introduce a new notation for this; perhaps:

$1 \boldsymbol{s}^{\prime \prime \prime}$. Adam is taller than Bilbo.

However, the problem is not one of notation, but one of understanding. The advocate of SSL needs to supply a semantics, which explains to the irrealist how a relation can "bridge the gulf between reality and fiction" without committing her to fictional objects. In particular, the SSL-advocate must offer a metalinguistic sentence which gives the truth-conditions of $(1)$, where that metalinguistic sentence does not entail (in the metalanguage) the existence of fictional objects. I intend to argue that this cannot be done to the irrealist's satisfaction.

A realist semantics. To show how things can go wrong, we shall start by considering a straightforward model-theoretic semantics for SSL. In a model-theoretic semantics, an interpretation of a (set of) SSL-sentence(s) would be given by a function, $\iota$, from names to objects within a domain, and from predicates to relations on that domain. In our particular case, a completely standard modeltheoretic semantics would tell us that $(1 \beta)$ is true iff: ${ }^{15}$

9. $\iota$ ("Adam") has the relation $\iota$ ("is taller than") to $\iota$ ("Bilbo")

Since we are assuming that $(1)$ is true, this would commit us to a domain which contains an object $\iota$ ("Bilbo"). That would be a referent for "Bilbo". And that would no longer be irrealism: it would be to say that fictional names name real objects after all, so long as the names are underlined. Indeed, a model-theoretic semantics turns SSL into a multi-sorted logic for realists about fictions, where underlining indicates the sortal (real or fictional) of an object.

This leads us to a First Moral. In any metalinguistic sentence (e.g. 9p) giving the truth-conditions of any SSL-sentence (e.g. (1)), any name of a fictional

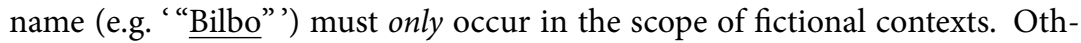
erwise, we will be committed in the metalanguage to the existence of fictional objects, and so irrealists will be unable to use SSL.

An incorporating-irrealist semantics. Evidently, underlined contexts must not be treated realistically, as they are in a straightforward model-theoretic semantics. Equally, non-underlined contexts must not be treated as part of some wider fiction. For example, suppose that we allow operators into our metalanguage. Then we might say that $(1 ;)$ is true iff

9. according to F $\{\iota$ ("Adam") has the relation $\iota$ ("is taller than") to $\iota$ ("Bilbo") $\}$ for some appropriate metalinguistic operator "according to F". On this account, then, there is a (metalinguistic) fiction, $\mathrm{F}$, according to which there is both a referent for "Adam" and a referent for "Bilbo". This is an extended (metalinguistic) 
fiction which contains both Adam and Bilbo. And that is just incorporatingirrealism in the metalanguage. But the whole point of SSL was to offer irrealists a new solution to the operator problem.

This leads us to a Second Moral. In any metalinguistic sentence (e.g. (9i)) giving the truth-conditions of any SSL-sentence (e.g. (1p)), any name of a name of a real object, (e.g. "Adam"') must not occur within the scope of any fictional context. After all, Adam is a real entity, hence putting "Adam"' within the scope of a fictional context would either make him fictional, or return us to incorporating-irrealism.

An operator problem in the metalanguage. The combined upshot of the two Morals is easy to see. Naïve model-theoretic semantics yields (9) as the metalinguistic sentence which expresses (11)'s truth-conditions. Although superficially complicated, (9) is really just a sentence of the form " $a R s$ s": ' $a$ ' is ' $\iota$ ("Adam")'; ' $b$ ' is ' $\iota$ ("Bilbo")'; and ' $R$ ' is 'has the relation $\iota$ ("is taller than") to'. But, by the First Moral, ' $\iota$ ("Bilbo")' can only occur in the scope of fictional contexts. And, by the Second Moral, ' $($ ('Adam")' must not occur in the scope of any fictional operator. In short: (9) is just a metalinguistic version of the operator problem.

In response, advocates of SSL will have look for a metalinguistic sentence which is different from both (9) and (9), but which is appropriately "nearby" to (9). Perhaps they will succeed in finding one. But if they do, they will simply have presented us with austere-irrealism in the metalanguage. Again, SSL will fail to offer irrealists any new solution to the operator problem.

\section{Spottiness all the way down?}

Sainsbury wanted to use SSL to solve the operator problem. We have seen that the operator problem arises in the metalanguage for SSL. The detour through SSL and metalinguistic-ascent seems to be an unnecessary shuffle. Surely this is the end for spotty scope.

In fact, there is a last-ditch reply. Someone who is truly convinced of the merits of SSL might think that the occurrence of the operator problem in the metalanguage indicates that spotty scope has to be a feature of the metalanguage too. Pursuing this line of thought, she might claim that $(1 ;)$ is true iff:

$$
\text { 9. } \iota \text { ("Adam") has the relation } \iota \text { ("is taller than") to } \iota \text { ("Bilbo") }
$$

The normal underlining in (9p) is a feature of the object language, whereas the wavy-underlining is a feature of the metalanguage. So the value of the interpretation function $\iota$ with argument "Bilbo" itself falls under metalinguistic spotty scope.

The operator problem will now, of course, recur in any metametalanguage in which we offer the truth-conditions for $(9 \mathrm{p})$ ). No matter-says our SSL-enthusiast-spotty scope occurs there too. And in the metametametalanguage. And so on, "all the way down". ${ }^{16}$ 
This response pushes us back to the question of why SSL needed a semantics in the first place. My initial concern with (1) was that is taller than has to straddle the gulf between fiction and reality: it is a relation which is somehow meant to hold between Adam and Bilbo, and I could not make sense of that. I asked for a semantics to explain this, and the advocate of SSL has presented me with (93). This tells me that some relation holds between $\iota$ ("Adam") and $\iota$ ("Bilbo"); namely the relation has the relation $\iota$ ("is taller than") to. But this relation must itself straddle the gulf between fiction and reality; between $\iota$ ("Adam"), who exists, and $\iota$ ("Bilbo"), who doesn't. If my initial concern was a legitimate concern, it has not been answered at all.

Our SSL-enthusiast must, then, believe that my initial concern is illegitimate. She must think that spotty scope is an utterly primitive feature of language (in general) which cannot be further explained. She ought to have rejected my demand to give a semantics for those relations which bridge between world and fiction. She should simply have said: If you don't understand now, you never will.

At this point, we need to take stock. The operator problem arose because irrealists wanted to treat sentences like (1)-(6) as true, but they did not want to have to believe in fictional objects, like Bilbo Baggins. Sentences like (1) - 6) seem to force us to believe in fictional objects just because they seem to involve talking about fictional objects. So, these sentences threaten to turn us into realists about fictions just because we have accepted that to be the value of a variable entails existence. ${ }^{17}$

The SSL-enthusiast, though, has told us that she can use underlined names without incurring ontological commitment. Since it is clear that one can't make a problem go away just by underlining it, the irrealist must just be rejecting our criterion for ontological commitment. Perhaps the SSL-advocate is right to do this; perhaps not. But what is clear is that the SSL-advocate is no longer engaged in the metaphysical debate between realists and irrealists. Rather, she is engaged in a metametaphysical debate. She disagrees with both the realist and the irrealist about what it takes to believe in fictional objects. So, she cannot claim to be offering an irrealist solution to the operator problem. Rather, she is attacking the very terms of the debate which give rise to the operator problem.

Whether or not SSL can be harnessed by metametaphysicians is a question I cannot pursue here. But either way, I have established what I wanted to show: SSL cannot offer the irrealist with a novel solution to the operator problem.

\section{Generalising from the failure of spotty scope}

To close, I would like to consider the extent to which we can treat the failure of spotty scope as a case study. I want to suggest that any novel syntactic approach to the operator problem will face the same objections as I have raised against SSL.

Suppose we attempted to solve the operator problem by augmenting our syntax with some other device, such as Kaplan's monadic quantifying-out opera- 
tor, “ $\curvearrowleft$ ". ${ }^{18}$ Intuitively, this operator allows us to step back outside the context of the operator that we are in, whilst retaining semantic values from that context. Using this operator, we might treat (1) thus:

1.k. according to $\operatorname{Lot} R\{\exists x(x$ is a hobbit and $\curvearrowleft\{$ Adam is taller than $x\})\}$

The idea is that the formula " $\curvearrowleft\{$ Adam is taller than $x$ \}" is to be evaluated outside the scope of "according to LotR". The formal features associated with quantifyingout are, in a sense, much like the features of Sainsbury's spotty scope. Accordingly, the metaphysical problem will arise just as soon as we consider the semantics for contexts governed by “ $\curvearrowleft$ ”. (1 k) is to be rendered true by instantiating " $x$ " with "Bilbo", which is legitimate since the quantifier falls within the scope of the fictional operator. But, respecting the " $\curvearrowleft$ " operator, we must then evaluate "Adam is taller than Bilbo" outside the context of LotR. How are we to do this, unless we are realists about Bilbo? That was precisely the original problem.

Any novel syntactic device will face this problem. What this shows is that the operator problem is not really a problem concerning syntactic scope. It is a metaphysical problem. Brutally, our question is: How can a relation obtain when one of the relata doesn't exist? ${ }^{19}$

I think the obvious answer is the correct one: It can't, and no amount of logical tinkering will show otherwise.

\section{Appendix: Hintikka semantics for quantifiers}

In this Appendix, I sketch a Hintikka semantics for BQL, as discussed in $\$ 4$ This sketch follows the much more detailed explanations given by Hintikka (1968. pp. 55-7), (1974 pp. 156ff) and Forster (2006). I shall deal only with the semantics for quantifiers; the semantics can easily be extended to handle propositional connectives, but we do not need to consider this extension.

We start with the case of ordinary (linear) first-order logic. Two players, Abelard and Eloïse, are playing a game of perfect information, using closed prenex sentences of first-order logic. They play by instantiating quantifiers in the sentence. Abelard instantiates all the universal quantifiers; Eloïse instantiates all the existential quantifiers; and they take their turns at instantiating quantifiers in the order that the quantifiers occur in the sentence. The result is a quantifier-free formula, $\varphi$, together with an interpretation, $\iota$, for every free variable in $\varphi$. Abelard wins the game if $\iota$ makes $\varphi$ false; Eloïse wins if $\iota$ makes $\varphi$ true.

We say that Eloïse has a winning strategy for a sentence iff Eloïse can win on that sentence no matter how Abelard plays. It is easy to show that a sentence of first-order logic is true iff Eloïse has a winning strategy.

To extend the Hintikka game semantics to prenex sentences of BQL, we drop the assumption that the players have perfect information. This is most easily explained by replacing the individual players with teams of players: Team Abelard and Team Eloïse. Both teams have one player for each branch in the quantifier 
prefix. (Thus, in the case of (7p), Team Abelard comprises $A_{1}$ and $A_{2}$, and Team Eloïse comprises $\mathrm{E}_{1}$ and $\mathrm{E}_{2}$.)

To start the game, a prenex sentence of BQL is produced. The members of Team Eloïse get together, to decide upon a strategy. They then separate, and may not communicate again until the end of the game. On each branch of the quantifier prefix, play occurs just as it does in the case of standard first-order logic, although each player can only see what is happening on their line of the prefix. (Thus, in the case of (7p): $\mathrm{A}_{1}$ instantiates " $x$ " and $\mathrm{A}_{2}$ instantiates " $s$ "; $\mathrm{E}_{1}$ must instantiate " $y$ ", and can see what $\mathrm{A}_{1}$ does, but not what $\mathrm{A}_{2}$ or $\mathrm{E}_{2}$ do; $\mathrm{E}_{2}$ must instantiate " $t$ ", and can see what $\mathrm{A}_{2}$ does, but not what $\mathrm{A}_{1}$ or $\mathrm{E}_{1}$ do.) Again, the result is a quantifier-free formula, $\varphi$, together with an interpretation, $\iota$, which makes $\varphi$ true or false, and Team Eloïse wins iff $\iota$ makes $\varphi$ true.

We now stipulate that a sentence of BQL is true iff Team Eloïse has a winning strategy. This supplies a semantics for BQL.

Intuitively, the "spottiness" in BQL corresponds to the limited information available to the players during the game. The reason that BQL cannot help Sainsbury is that 11 is a BQL-sentence in which there are no moves for any players to make.

\section{Notes}

${ }^{1}$ This use of the word "irrealism" follows Sainsbury 2010). Note that noneists, like Priest (2005), are not irrealists: noneists think that some (non-existent) things are hobbits, and that we can refer to them.

${ }^{2}$ Sainsbury 2005 pp. 64-75) surveys several treatments.

${ }^{3}$ The problem is discussed in some form by Geach (1967), Howell (1979 pp. 151ff), Walton (1990 pp. 409-6), Vision (1993. pp. 150-2), Priest 2005 p. 123) and Sainsbury 2005 pp. 202-10), (2006), 2010 pp. 41-3, 122-5).

${ }^{4}$ Thanks to a referee for Noûs, who pointed out to me that the operator problem may arise for realists who think that Bilbo is an abstract artefact created by Tolkein. The problem also arises for noneists; see note 19

${ }^{5}$ Priest (2005 p. 123); see also Walton 1990 p. 413). This strategy is similar to one employed by Milne (1992) to handle transworld and transtemporal comparisons, such as: "Adam is taller than he might have been" and "Adam is taller than he was".

${ }^{6}$ Sainsbury 2005 pp. 208-15) advocated austere-irrealism with some reservations. But more recently, he has raised worries for austere-irrealism 2010 pp. 123). In essence, Sainsbury's worry is that there is no $x$ such that according to LotR \{Bilbo's height is exactly $x$ inches\}. This worry is misplaced. Since Adam is over 78 inches tall, all the austere-irrealist requires in order to assert something like [1] is that, according to LotR \{Bilbo's height is under 78 inches\}.

${ }^{7}$ Howell 1979 p. 152) raised example 6.

${ }^{8}$ Sainsbury (2010 pp. 122-5) states that incorporating-irrealism is preferable to austere-irrealism (see note 6. Walton's (1990) "unofficial" fictions are something like my extended fictions. Howell 1979. pp. 156ff) criticises incorporating-irrealism.

${ }^{9}$ Relatedly: some irrealists may attempt to solve the operator problem by claiming that they never sincerely assert operator problem style sentences, but merely pretend to assert them. I suspect, though, that this changes very little. To pretend is to adopt an internal attitude to an operator; for example, if we pretend that Bilbo exists, we simply act internally to the operator "according to extended-LotR". Consequently, pretence offers irrealists no distinctively different approach to the operator problem. (Pretence may, of course, offer plenty of distinctive approaches to other issues in the philosophy of fiction.)

${ }^{10}$ Sainsbury includes (underlined) prefixes to delimit the particular fiction in question, so his 
paraphrase of 11 would be something like "according to Lot R, Bilbo is a hobbit and Adam is taller than Bilbo". For perspicuity, I have dropped the underlined prefixes.

${ }^{11}$ Sainsbury 2006 pp. 17-8). The example is from Hintikka (1974 p. 167).

${ }^{12}$ BQL goes by other names, e.g. "FPO" (since stacking quantifiers on top of one another Partially Orders them) and "IFL" (since this logic is Independence-Friendly). I shall not pursue the question of whether BQL is a first-order logic, in any interesting sense.

${ }^{13}$ Sainsbury 2006 p. 21 ).

${ }^{14}$ Thanks to Ioannis Votsis and Peter Godfrey-Smith for pressing me on this.

${ }^{15}$ More formally, but less perspicuously: $\langle\iota$ ("Adam"), $\iota($ "Bilbo") $\rangle \in \iota$ ("is taller than").

${ }^{16}$ Thanks to Philip Keller for suggesting this.

${ }^{17}$ Compare Quine's 1948 criterion for ontological commitment.

${ }^{18}$ Howell (1979 pp. 162-9), considers this and, like me, rejects it.

${ }^{19}$ This might suggest that the problem does not arise for noneists, who can simply offer " $\mathfrak{S} x \mathfrak{S} y(\mathrm{E} x \wedge$ $\neg \mathrm{E} y \wedge R x y$ )", where " $\mathfrak{S}$ " is the noneist's particular quantifier and " $\mathrm{E}$ " is a privileged predicate satisfied exactly by the existing things (see Priest (2005)). As mentioned in note 1 noneists are realists, so I have not considered how the operator problem affects them. As it happens, though, noneists face versions of the operator problem. First, problems arise if the relation in question entails existence. For example, Priest 2005 p. 123) thinks that having a height entails existence, so he handles 1 using a noneist version of $(1 \mathrm{l})$. Second, the operator problem arises if the noneist wants to explain how a relation could obtain when one of the relata not only fails to exist, but simply isn't (i.e. the relatum fails to fall under the noneist's particular quantifier).

\section{References}

Forster, Thomas (2006). "Deterministic and Nondeterministic Strategies for Hintikka games in First-order and Branching-quantifier logic". Logique et Analyse, 195, pp. 265-9.

GeACH, Peter (1967). "Intentional Identity”. The Journal of Philosophy, 64.20, pp. 627-32.

HiNTiKKA, Jaakko (1968). "Language-Games for Quantifiers". In REscher, Nicholas, editor: Studies in Logical Theory. Volume 2, Oxford: Basil Blackwell, pp. 46-72.

HintıkкA, Jaakko (1974). “Quantifiers vs. Quantificational Theory”. Linguistic Inquiry, 5, pp. 153-77.

Howell, Robert (1979). “Fictional Objects: How they Are and How they Aren't”. Poetics, 8, pp. 129-177.

Milne, Peter (1992). "Modal Metaphysics and Comparatives". Australasian Journal of Philosophy, 70.3, pp. 248-62.

Priest, Graham (2005). Towards Non-Being. Oxford: Clarendon Press.

Quine, Willard van Orman (1948). “On What There Is”. Review of Metaphysics, 2.5, pp. 21-36.

SAINSBURY, Roger Mark (2005). Reference without Referents. Oxford: Clarendon Press.

SAINSBURY, Roger Mark (2006). “Spotty Scope”. Analysis, 66.1, pp. 17-22.

SAINSBURY, Roger Mark (2010). Fiction and fictionalism. Oxfordshire: Routledge.

VIsion, Gerald (1993). "Fiction and Fictionalist Reductions". Pacific Philosophical Quarterly, 74.2, pp. 150-74.

Walton, Kendall L. (1990). Mimesis as Make-Believe: on the Foundations of the Representational Arts. London: Harvard University Press. 